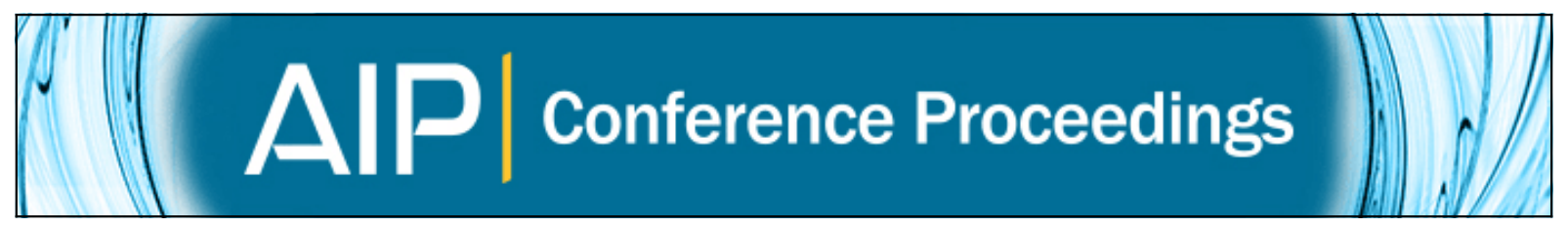

\title{
Adaptive and context-aware detection and classification of potential QoS degradation events in biomedical wireless sensor networks
}

Carlos Abreu, Francisco Miranda, and Paulo M. Mendes

Citation: AIP Conference Proceedings 1738, 370012 (2016); doi: 10.1063/1.4952157

View online: http://dx.doi.org/10.1063/1.4952157

View Table of Contents: http://scitation.aip.org/content/aip/proceeding/aipcp/1738?ver=pdfcov

Published by the AIP Publishing

\section{Articles you may be interested in}

Mixed integer nonlinear programming model of wireless pricing scheme with QoS attribute of bandwidth and endto-end delay

AIP Conf. Proc. 1705, 020050 (2016); 10.1063/1.4940298

Management challenges of context-aware learning

AIP Conf. Proc. 1644, 207 (2015); 10.1063/1.4907838

Energy Aware Clustering Algorithms for Wireless Sensor Networks

AIP Conf. Proc. 1389, 1110 (2011); 10.1063/1.3637807

Dynamic QoS Provisioning for Ethernet-based Networks

AIP Conf. Proc. 1060, 151 (2008); 10.1063/1.3037040

Quality of service (QoS) on public telephonic networks for multimedia transmission systems

J. Acoust. Soc. Am. 112, 2279 (2002); 10.1121/1.4779141 


\title{
Adaptive and Context-Aware Detection and Classification of Potential QoS Degradation Events in Biomedical Wireless Sensor Networks
}

\author{
Carlos Abreu ${ }^{*, \dagger}$, Francisco Miranda*,** and Paulo M. Mendes ${ }^{\dagger}$ \\ *Instituto Politécnico de Viana do Castelo, Viana do Castelo, Portugal \\ ${ }^{\dagger}$ CMEMS-UMINHO, Universidade do Minho, Braga, Portugal \\ ${ }^{* *}$ CIDMA, Universidade de Aveiro, Aveiro, Portugal
}

\begin{abstract}
The use of wireless sensor networks in healthcare has the potential to enhance the services provided to citizens. In particular, they play an important role in the development of state-of-the-art patient monitoring applications. Nevertheless, due to the critical nature of the data conveyed by such patient monitoring applications, they have to fulfil high standards of quality of service in order to obtain the confidence of all players in the healthcare industry. In such context, vis-à-vis the quality of service being provided by the wireless sensor network, this work presents an adaptive and context-aware method to detect and classify performance degradation events. The proposed method has the ability to catch the most significant and damaging variations on the metrics being used to quantify the quality of service provided by the network without overreacting to small and innocuous variations on the metric's value.
\end{abstract}

Keywords: Quality of service, adaptive, context-aware, biomedical wireless sensor networks.

PACS: $89.20 . \mathrm{Ff}$

\section{INTRODUCTION}

Biomedical Wireless Sensor Networks (BWSN) are characterised by a need for reliable and timely data delivery. Moreover, BWSN have to carry distinct data flows, each one having its own Quality of Service (QoS) requirements. In addition, BWSN should be autonomous, scalable, and operate for a long period without human intervention. In other words, they must fulfil a very demanding set of QoS requirements [1].

Furthermore, BWSN designed to support patient monitoring applications inside hospital facilities have to operate in harsh environments, regarding radio-frequency communications. Such adversities result from both, structural factors (e.g., metal doors and furniture or radiation shields) and interferences from other devices (e.g., Wi-Fi and Bluetooth networks). To further worsen this scenario, by being low-power wireless networks, BWSN are highly vulnerable to obstacles and human bodies [2, 3]. In fact, Ko et al. confirmed that the packet losses for IEEE 802.15.4-based networks are higher in hospitals than in other indoor environments [4].

From the previous discussion, it becomes crystal clear that the BWSN used to support the communications in patient monitoring applications must be permanently monitored in order to assess its performance and, if necessary, fire alerts to inform the network manager about the network status. In such context, Abreu et al. have proposed an adaptive and context-aware method to monitor the on-the-fly QoS provided by the BWSN [1]. The proposed method detects potential QoS degradation events by analysing the behaviour of each metric used to assess and quantify the QoS being provided by the network.

Carrying on the work presented in [1], this paper contributes with a method to classify the potential QoS degradation events detected by the QoS monitoring system, avoiding it from overreacting to minor changes on the network performance, while catching the most significant and damaging variations.

\section{DETECTION AND CLASSIFICATION OF POTENTIAL QOS DEGRADATION EVENTS}

The QoS monitoring module presented in [1] uses a set of dynamic metrics to assess the QoS provided by the BWSN. Those metrics are seen as time-domain functions modelled as:

$$
m(t)=m^{*}(t)+w g n(t), t \geq 0,
$$


where $m(t)$ represents the measured value of the metric, $m^{*}(t)$ signifies the real value of the metric, and the white Gaussian noise function $w g n(t)$ represents the metric's variations due to random interferences and the natural fluctuations of the network.

The estimated value of the metric is computed as:

$$
\hat{m}(n)=f(m, N, n)
$$

The $f(g, N, n)$ represents a moving average filter defined as:

$$
f(g, N, n)=\frac{1}{k} \sum_{i=0}^{k-1} g(n-i),
$$

where $g(\cdot)$ represents the signal to be filtered, $N$ is the number of observations used in the moving average, $n$ is the index of the most recent observation of the sample to be processed, and $k=n$ if $n<N$ or $k=N$ in other cases. The use of moving average filters reduces the effects of random instabilities on the metrics.

QoS degradation events are detected by looking for the dynamic of the metric being analysed, which is represented by its first derivative and denoted as $\dot{\hat{m}}(n)$. Therefore, two features were proposed to detect changes in the metric's value, namely the energy of $\dot{\hat{m}}(n)$ :

$$
E_{\dot{\hat{m}}}(n)=\sum_{i=0}^{k-1}|\dot{\hat{m}}(n-i)|^{2},
$$

and an energy threshold dynamically computed as:

$$
E_{\text {threshold }}(n)=f\left(E_{\dot{\hat{m}}}, N, n\right) .
$$

Changes on the network's performance are detected by comparing the actual $E_{\dot{\hat{m}}}$ against the $E_{\text {threshold }}$. In order to quantify such changes, potentially harmful to the network, a Metric Degradation Index (MDI) was defined as:

$$
\operatorname{MDI}(n)=\left\lfloor E_{\dot{\hat{m}}}(n) / E_{\text {threshold }}(n)\right\rfloor .
$$

The MDI gives quantitative information about the changes on the metric's energy.

By using this method, and depending on the value of the $E_{\text {threshold, }}$, very small variations on the metric's value can be detected. However, this could cause the QoS monitoring system to overreact, firing undesirable alerts. To avoid this, an adaptive and context-aware threshold is defined for the MDI as:

$$
\operatorname{MDI}_{\text {threshold }}(n)=\frac{1}{k^{*}} \sum_{j \in K} \operatorname{MDI}(n-j),
$$

where $K$ is the set of all values of $i \in\{0,1, \ldots, k-1\}$ such that $M D I(n-i)>M D I_{\text {threshold }}(n-1) * \alpha, k^{*}$ is the number of elements of $K$, and $\alpha \in[0,1]$. By using this threshold the QoS monitoring system is able to ignore small variations on the metric's value, avoiding the QoS monitoring system from overreacting. The coefficient $\alpha$ can be used to tune the response of the filter.

Applying the filter defined in (1), the QoS degradation events are fired only if the following condition is verified:

$$
M D I>M D I_{\text {threshold }}
$$

\section{EXPERIMENTAL RESULTS}

To assess the proposed classification method, a case study bearing in mind a typical BWSN is considered. During the development phase, the method was evaluated using a BWSN implemented in the Contiki OS [5] and simulated using the hardware emulator and network simulator tool known as COOJA [6]. Then, the suggested method was tested in a real hospital environment, see Fig.1. The experiments were made in a small-sized hospital to be found in Esposende, a small city nearby Braga, Portugal; the hospital is known as "Hospital Valentim Ribeiro".

The scenario presented in the Fig.1 was assessed with and without the proposed classification method (i.e., the $M D I_{\text {threshold }}$ ). When applying the proposed classification method, three values were considered for the coefficient $\alpha$, 


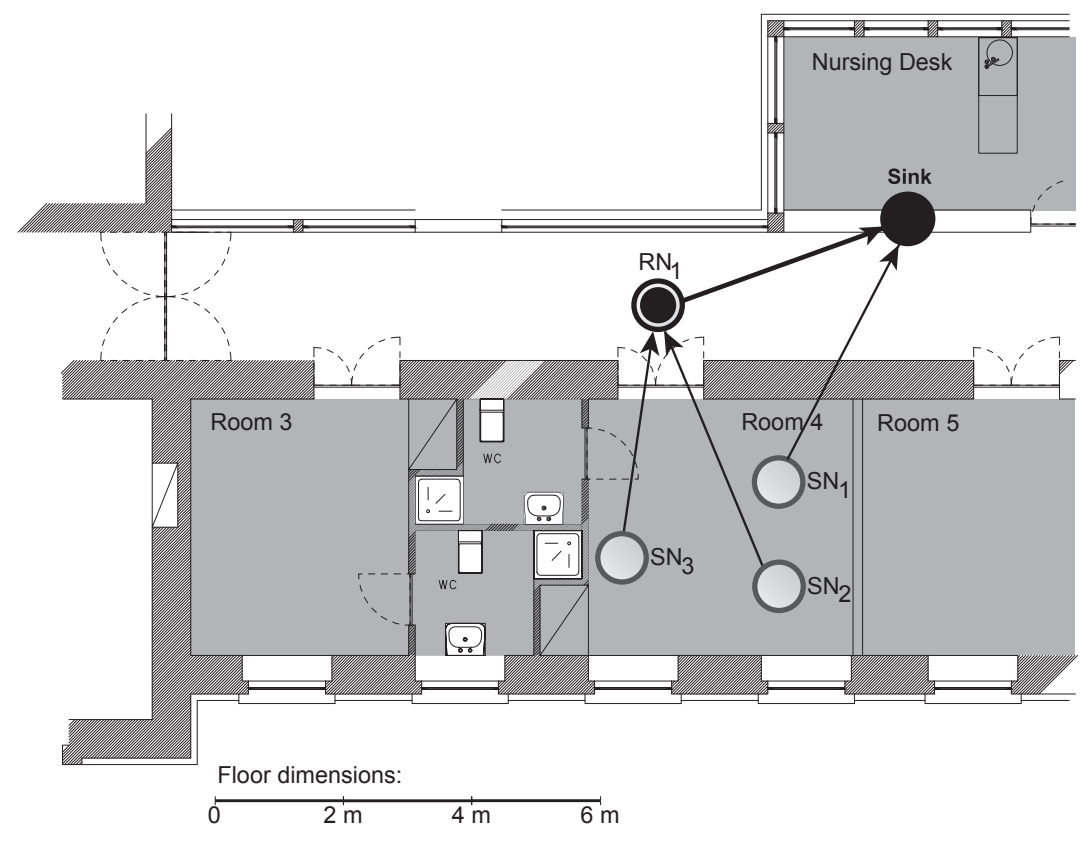

FIGURE 1. BWSN used to assess the proposed adaptive and context-aware classification method.

i.e., $\alpha=0.2, \alpha=0.4$ and $\alpha=0.6$. In all experiments, the metric used as a figure of merit to quantify the QoS provided by the BWSN was the Packet Reception Ratio (PRR).

The results obtained show that the number of QoS degradation events is significantly reduced when using the suggested classification method, see Fig.2. If no threshold is used, the detection algorithm fires 20 QoS degradation events, see Fig.2 a). Although, when using the proposed $M D I_{\text {threshold }}$ (1), the decision condition (2), and depending on the value of the coefficient $\alpha$, the number of QoS degradation events can be reduced, while getting the most relevant variations on the PRR.

As presented in the Fig.2 b), the number of QoS events fired when using the suggested classification method with $\alpha=0.2$ is 15 which corresponds to a reduction of about $25 \%$ when comparing with the results obtained in the Fig.2 a). On its turn, the Fig. 2 c) shows the results found when using $\alpha=0.4$. In this case, the number of QoS degradation events fired is 10 , resulting in a decrease of about $50 \%$. Finally, by using $\alpha=0.6$, only 7 events are fired, corresponding to a reduction of about $65 \%$, see Fig. 2 d). It is important to notice that the classification method proposed in this work does not affect the ability of the QoS monitoring system presented in [1] to detect potential harmful variations on the QoS being provided by the network.

\section{CONCLUSION}

Due to the challenging requirements of healthcare services and applications, biomedical wireless sensor networks have to fulfil high standards of quality of service. Therefore, we presented an adaptive and context-aware detection and classification method, to be used by the QoS based management system presented in [1]. The proposed method greatly reduces the number of quality of service degradation events fired while catching the most significant, and harmful, variations on the metric being used to measure the quality of service provided by the network.

\section{ACKNOWLEDGMENTS}

This work has been supported by the PhD grant of Portuguese Foundation for Science and Technology (FCT), SFRH/BD/61278/2009. Miranda was also supported by Portuguese funds through the CIDMA - Center for Research and Development in Mathematics and Applications, and the FCT. 


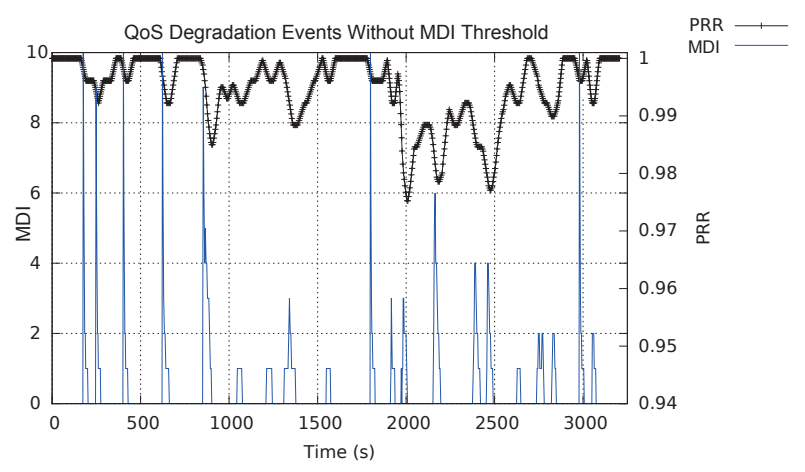

(a)

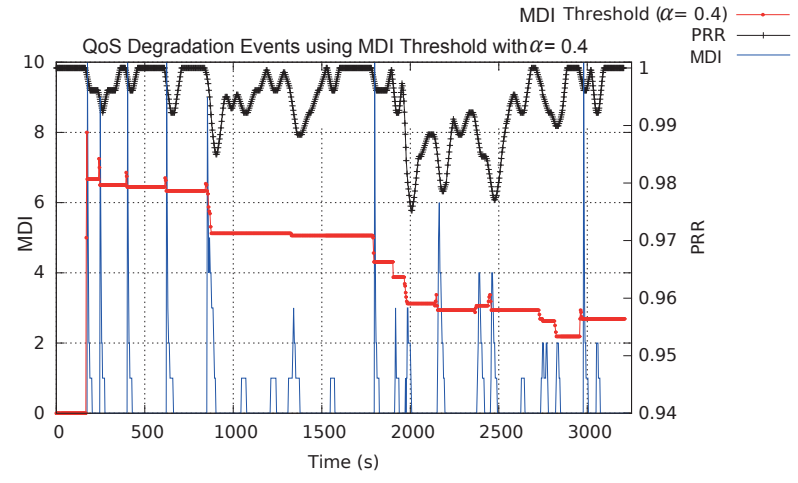

(c)
MDI Threshold $(\alpha=0.2)$

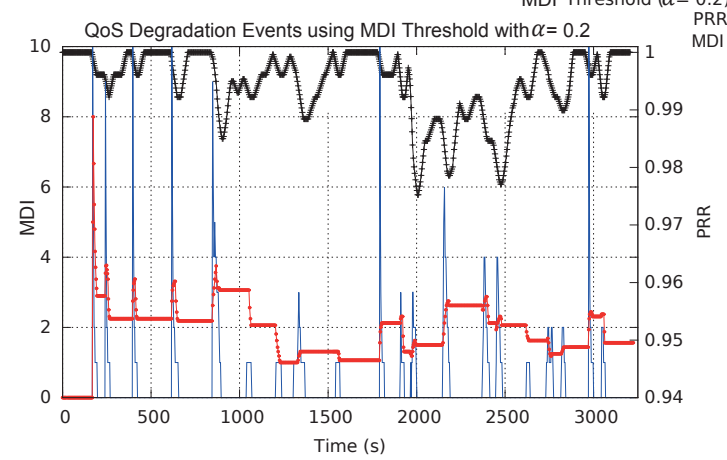

(b)

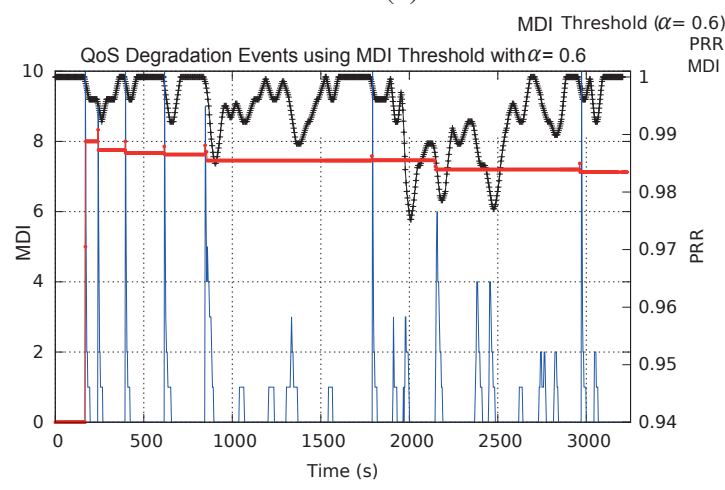

(d)

FIGURE 2. Results obtained when assessing the proposed classification method.

\section{REFERENCES}

1. C. Abreu, F. Miranda, M. Ricardo, and P. M. Mendes, "QoS-Based Management of Biomedical Wireless Sensor Networks for Patient Monitoring," SpringerPlus 3(239), 13 pages (2014).

2. JG. Ko, C. Lu, M. B. Srivastava, J. A. Stankovic, A. Terzis, and M. Welsh, "Wireless Sensor Networks for Healthcare," Proceedings of the IEEE 98(11), 1947-1960 (2010).

3. M. Chen, S. Gonzalez, A. Vasilakos, H. Cao, and V. C. M. Leung, "Body Area Networks: A Survey," Mobile Networks and Applications 16(2), 171-193 (2011).

4. JG. Ko, T. Gao, and A. Terzis, "Empirical Study of a Medical Sensor Application in an Urban Emergency Department," in Proceedings of the Fourth International Conference on Body Area Networks, ICST, Brussels, Belgium, 2009, Article No. 10.

5. A. Dunkels, B. Gronvall, and T. Voigt, "Contiki - A Lightweight and Flexible Operating System for Tiny Networked Sensors," in Proceedings of the 29th Annual IEEE International Conference on Local Computer Networks, Tampa, FL, USA, 2004, pp. 455-462.

6. F. Osterlind, A. Dunkels, J. Eriksson, N. Finne, and T. Voigt, "Cross-Level Sensor Network Simulation with COOJA," in Proceedings of the 31st IEEE Conference on Local Computer Networks, Tampa, FL, USA, 2006, pp. 641-648. 